\title{
TRAIL Promotes Epithelial-to-mesenchymal Transition by Inducing PD-L1 Expression in Esophageal Squamous Cell Carcinomas
}

\section{Huanyu Zhang}

Zhengzhou University First Affiliated Hospital

\section{Guohui Qin}

Zhengzhou University First Affiliated Hospital

Huiyun Yang

Zhengzhou University First Affiliated Hospital

Jinyan Liu

Zhengzhou University First Affiliated Hospital

\section{Peng Wu}

Zhengzhou University First Affiliated Hospital

\section{Hongwei Hu}

Zhengzhou University First Affiliated Hospital

\section{Shasha Liu}

Zhengzhou University First Affiliated Hospital

\section{Li Yang}

Zhengzhou University First Affiliated Hospital

\section{Yi Zhang ( $\nabla$ yizhang@zzu.edu.cn )}

The First Affiliated Hospital of Zhengzhou University https://orcid.org/0000-0001-9861-4681

\section{Research}

Keywords: TRAIL, tumorigenesis, EMT, PD-L1, Immunotherapy

Posted Date: December 3rd, 2020

DOI: https://doi.org/10.21203/rs.3.rs-117700/v1

License: (c) (1) This work is licensed under a Creative Commons Attribution 4.0 International License. Read Full License 


\section{Abstract}

Background: Tumor necrosis factor-associated apoptosis-inducing ligand (TRAIL) was initially considered an immunity guard; however, its function remains controversial. Besides immune cells, lung and colon cancer cells have also been reported to express TRAIL, which can promote tumor invasion and metastasis. However, the biological function and underlying mechanism of action of TRAIL in esophageal squamous cell carcinoma (ESCC) remain poorly elucidated.

Methods: The ESCC cells stemness, migration, and proliferation ability was assessed by sphere formation, Transwell, and CCK8 assay. The stemness- and EMT- related genes expression levels were analyzed by Western blot and RT-qPCR. The signal activation was conducted by Western blot. The PDX Model were performed to confirm our findings in vitro.

Results: Herein, we found that TRAIL is a negative predictor in patients with ESCC. To further investigate the biological function of TRAIL, we established TRAIL knockdown and overexpression ESCC cell lines and found that TRAIL induced epithelial-mesenchymal transition (EMT) and promoted tumor aggressiveness. Furthermore, we demonstrated that TRAIL- overexpressing cells upregulated PD-L1 expression, which was dependent on the p-ERK/STAT3 signaling pathway. We obtained similar results when using recombinant human TRAIL. Finally, we validated the biological role and mechanism of action of TRAIL in vivo.

Conclusions: These findings demonstrate that TRAIL promotes ESCC progression by enhancing PD-L1 expression, which induces EMT. This may explain the failure of TRAIL preclinical trials.

Financial support: This work was supported by the National Key Research and Development (2018YFC1313400), the National Nature Science Foundation of China (U1804281, 91942314) and the National Science Fund for Distinguished Young Scholars (82001659).

\section{Introduction}

Tumor necrosis factor-related apoptosis inducing ligand (TRAIL) is a member of the tumor necrosis factor superfamily (TNFSF) and is encoded by the gene TNFSF10. To function, TRAIL must interact with its receptors. To date, five TRAIL receptors have been identified: TRAIL-R1 (DR4), TRAIL-R2 (DR5), TRAILR3 (DCR4), TRAIL-R4 (DCR5), and osteoprotegerin (OPG)[1-3]. Initially, TRAIL was found expressed on various immune cells, such as T cells, B cells, and NK cells, and involved in both innate and adaptive immune responses[4-6]. Until recently, TRAIL shows a diversity of function in the tumor microenvironment. On the one hand, TRAIL could target tumor cells and promote apoptosis via binding to its receptors DR4/5[7, 8]. On the other hand, tumor cells could recruit M2 by expressing TRAIL, remodeling the tumor microenvironment and promoting tumor progression[9]. However, how TRAIL regulates tumor cell epithelial-to-mesenchymal transition (EMT) to affect tumor cell metastasis has not been investigated. 
Tumor invasion and metastasis is a complex multi-step cascade of responses that is constantly changing by the interaction of tumor cells with the host microenvironment[10], and they are the main reasons for the poor prognosis and low survival rate in the clinic. Among the various factors, such as inflammatory factors and immunosuppressive cells, epithelial-to-mesenchymal transition is considered the main contributor to distant metastasis[10,11]. Many factors have been reported to induce EMT in a variety of cancers. Recently, the correlation between PD-L1 expression and EMT has attracted increasing attention[12]. It has been demonstrated that PD-L1 promotes EMT in esophageal cancer, pancreatic cancer, breast cancer, and non-small cell lung cancers, leading to tumor progression[13-16]. Meanwhile, it has been reported that TRAIL and PD-L1 can be expressed simultaneously on tumor cells[17]. However, factors inducing the abnormal expression of PD-L1 are less defined. Esophageal squamous cell carcinoma (ESCC) is one of the most aggressive malignant tumors, and with various therapies, the 5-year survival rate of ESCC is only $15-25 \%[18,19]$. Therefore, revealing the underlying mechanism of ESCC distant metastasis may be fundamental for elevating clinical efficacy, but studies on blocking EMT to inhibit ESCC progression currently lack effective targets.

In this study, we found that TRAIL was a poor predictor in patients with ESCC. To further explain the phenomenon, we explored the source of TRAIL in the ESCC tumor microenvironment and evaluated its function in vitro and in vivo. We found that TRAIL was expressed by ESCC cells and can induce EMT by regulating PD-L1 expression. Knocking down TRAIL (TNFSF10) in ESCC cells suppressed EMT through inhibition of ERK/STAT3 signaling. It is evident that TRAIL can promote ESCC progression by influencing EMT, thus TRAIL has the potential to act as a key molecule to target EMT and inhibit ESCC progression in the future.

\section{Materials And Methods}

\section{Human clinical samples}

A total of 83 paired tumor and corresponding nontumor tissues (located more than $3 \mathrm{~cm}$ away from tumor tissues) were freshly obtained from patients with ESCC (diagnosed in 2017-2018) at the department of Thoracic Surgery of the First Affiliated Hospital of Zhengzhou University (Zhengzhou, China). All patients provided written informed consent before tissues or associated clinical information were collected, and this research was approved by the Institutional Ethical Committee of the First Affiliated Hospital of Zhengzhou University (2018-KY-92). Written informed consent was obtained from each patient with available follow-up information prior to participation.

\section{Cell lines and cell culture}

Human ESCC cell lines (KYSE150, KYSE70, EC1, EC109, EC9706, TE1, and TE7), and human embryonic kidney epithelial 293T cells (293T) were obtained from the Cell bank Shanghai Institutes for Biological Sciences of the Chinese Academy of Sciences. ESCC cells were cultured in RPMI 1640 medium supplemented with $10 \%$ FBS and $1 \%$ cyan streptomycin mixture. The virus packaging cell line 293T 
cultured in DMEM supplemented with 10\% FBS and 1\% cyan streptomycin mixture. Cells were cultured in an incubator with $5 \% \mathrm{CO}_{2}$ and $37^{\circ} \mathrm{C}$.

\section{Cell proliferation assays}

A total of $1 \times 10^{3}$ cells per well were plated in 96-well plates for cell proliferation assays. We set up five replicate wells for each group per experiment. Each experiment was repeated three times. CCK-8 (10 $\mu \mathrm{L}$; CK04-500; Dojindo, Kumamoto, Japan) was added at 0, 24, 48, and $96 \mathrm{~h}$ and incubated at $37^{\circ} \mathrm{C}$ for $2 \mathrm{~h}$. Absorbance was detected at $450 \mathrm{~nm}$ using an enzyme-labeled instrument (Multiskan MK3; Thermo Fisher Scientific, Waltham, MA, USA).

\section{Transwell invasion assays}

A Transwell plate with an $8 \mu \mathrm{m}$ membrane was used for testing tumor cell migration. Tumor cells $(1 \times$ $10^{5}$ ) without resuspension in serum were inoculated into the upper chamber, and $600 \mu \mathrm{L}$ of complete medium was added to the lower chamber. The cells were cultured in a cell incubator for $24 \mathrm{~h}$, fixed with $4 \%$ paraformaldehyde at room temperature for $30 \mathrm{~min}$, and stained with $0.1 \%$ crystal violet solution for 30 min. After taking pictures, the number of cells in each field was counted and a statistical chart was drawn.

\section{Cell sphere experiment}

The cell density was adjusted to $2 \times 10^{3}$ cells $/ \mathrm{mL}$, after which a $2-\mathrm{mL}$ cell suspension was added to a 24well low adhesion plate. After 5 days, the cell spheres were observed and counted under a microscope.

\section{RNA isolation and real-time PCR}

Total RNA was isolated using RNAiso Plus (Takara Bio, Shiga, Japan) and reverse-transcribed using PrimeScript $^{\mathrm{TM}}$ II 1st Strand cDNA Synthesis Kit (Takara Bio) according to manufacturer's instructions. RTqPCR was performed using SYBR Green (BCS, Australia). GAPDH was used for normalization of data. The data were analyzed using the $2^{\Delta \Delta \mathrm{Ct}}$ method. All primers are listed in Supplementary Table 1.

\section{Western blotting}

Protein samples were isolated from cells and animal tissues, after which they were resolved by SDSpolyacrylamide gel electrophoresis and transferred to a 0.2- $\mu \mathrm{m}$ nitrocellulose membrane (GE Healthcare, Chicago, IL USA). The blots were blocked with $5 \%$ non-fat dry milk in Tris-buffered saline for $2 \mathrm{~h}$ at room temperature and immunoblotted with the appropriate primary antibody at $4^{\circ} \mathrm{C}$ overnight. The next day, the blots were incubated for $1 \mathrm{~h}$ at room temperature with secondary antibodies. The Fusion FX7 ECL western blot system (Vilber Lourmat, Marne-la-Vallée, France) was used to visualize the protein signals. All antibodies are listed in Supplementary Table 1.

\section{Immunohistochemistry}

Tissue paraffin sections of tumors were deparaffinized in xylene and rehydrated. Slides were blocked and incubated with primary antibody at $4{ }^{\circ} \mathrm{C}$ overnight. The next day, slides were treated with biotinylated IgG 
secondary antibodies, followed by detection with the DAB substrate kit (Zsbio, Beijing, China). Images were acquired using a microscope (Olympus, IX73). Staining was evaluated based on intensity (negative $=0$; weak $=1$; moderate $=2$; and high $=3)$ of immunostaining and density $(0 \%=0 ; 1-40 \%=1 ; 41-75 \%=$ $2 ;>76 \%=3$ ) of positive tumor cells. All antibodies are listed in Supplementary Table 1.

\section{Immunofluorescence}

Cells $\left(5 \times 10^{4}\right)$ were seeded into 24 -well plates, fixed in $4 \%$ paraformaldehyde, and permeabilized with $0.1 \%$ Triton X-100. Next, $5 \%$ goat serum was used to block cells for 30 min, after which the cells were incubated with primary antibodies at $4{ }^{\circ} \mathrm{C}$ overnight. The cells were then incubated with secondary antibodies (BioLegend, San Diego, CA) for $1 \mathrm{~h}$ and counterstained with 4',6-diamidino-2-phenylindole (DAPl; BioLegend). Images were acquired using a fluorescence microscope and analyzed using ImageJ software (National Institutes of Health, Bethesda, MD). All antibodies are listed in Supplementary Table 1.

\section{Plasmid construction and stable transfection}

The hU6-MCS-Ubiquitin-EGFP-IRES-puromycin plasmids were purchased from GenePharma (Shanghai, China). High-titer lentivirus was packaged in HEK293T cells using transfection kits (Polyplus transfection \#20Y0305L9). Viral supernatants were collected at $48 \mathrm{~h}$ after transfection, filtered, and added to ESCC cells in the presence of $10 \mu \mathrm{g} / \mathrm{mL}$ polybrene for $24 \mathrm{~h}$. Finally, the transfection efficiency was checked by RT-qPCR and flow cytometer.

\section{siRNA transfection}

The cells were cultured in 6 -well plates at $3 \times 10^{5}$ cells per well. The total amount of transfection mixture for each well consisted of $200 \mu \mathrm{L}$ jetPRIME buffer, $10 \mu \mathrm{L}$ siRNA, $4 \mu \mathrm{L}$ jetPRIME reagent. The mixture was then centrifuged with a palm centrifuge and incubated at room temperature for $15 \mathrm{~min}$. The above transfection mixture system was evenly added into a hole. After $60 \mathrm{~h}$, the cells were collected for subsequent experiments.

\section{Flow cytometry analysis}

Cells $\left(1 \times 10^{6}\right)$ were stained (specific antibody information is listed in Table 1$)$, vortexed, and incubated in a refrigerator at $4{ }^{\circ} \mathrm{C}$ in the dark for $15 \mathrm{~min}$. After washing with $1 \mathrm{~mL}$ flow buffer (PBS with $2 \%$ serum), the samples were analyzed using a BDFACS Canto II Cell Analyzer (BD Biosciences, Franklin Lakes, NJ). FlowJo software (TreeStar, Inc., Ashland, OR) was used for data analysis.

\section{Animal experiments}

Female athymic BALB/c nude mice (4-6 weeks of age) were purchased from Beijing Vital River Laboratory Animal Technology Company (Beijing, China). All animal procedures were conducted in accordance with the Guide for the Care and Use of Laboratory Animals and were approved by the Institutional Animal Care and Use Committee of the First Affiliated Hospital of Zhengzhou University. After the mice were randomly separated into groups, each mouse was subcutaneously injected with $5 \times$ $10^{6}$ tumor cells. After 21 days, the mice were sacrificed. Tumor size was measured using calipers every 
two days and tumor volume was calculated using the formula $V=\left(\right.$ width $^{2} \times$ length $) / 2$. All the xenograft mouse model experiments were conducted in the Henan Key Laboratory for Pharmacology of Liver Diseases, and the animals' certificate were approved by the ethics committee of Henan Key Laboratory for Pharmacology of Liver Diseases (Approval No. 2019-41).

\section{The Cancer Genome Atlas (TCGA) database analysis}

mRNA sequencing data of patients with ESCC and healthy individuals were downloaded from TCGA database. Fifteen immunosuppressive molecules, such as PD-L1 (CD274), were selected for correlation analysis with TRAIL. Patients with ESCC were divided into TRAIL ${ }^{\text {high }}$ and TRAIL ${ }^{\text {low }}$ groups according to TRAIL mRNA expression.

\section{Statistical analysis}

For experiments with two groups, statistical significance was determined by Student's $t$-test. For experiments with more than three groups, analysis of variance followed by post-hoc pairwise comparisons was performed. Kaplan-Meier analysis was used to determine the difference in overall survival. Data shown are the means \pm SD of at least three independent experiments performed in duplicate. $P$ values $<0.05$ were considered statistically significant.

\section{Results}

\section{Abnormal expression of TRAIL in ESCC is negatively correlated with patient clinical outcomes}

To reveal the expression patterns of inflammatory factors and the corresponding receptors in ESCC tumor and normal tissues, we analyzed TCGA RNA-seq database. We found that CXCL14, TGF $\beta 1$, and TRAIL (TNFSF10) were highly expressed in tumor tissues compared with adjacent normal samples (Fig. 1a). Next, we analyzed the relationship between these three genes and patient clinical parameters. CXCL14 and TGF $\beta 1$ were not correlated with lymph node metastasis, staging, or overall survival (Fig. 1b, c). Only TRAIL (TNFSF10) had a significant positive relationship with lymph node metastasis and staging, and patients with high TRAIL (TNFSF10) levels exhibited poor prognosis (Fig. 1d). To further verify this, we analyzed TRAIL expression in 83 patients with ESCC at both the mRNA and protein levels. A similar trend was observed, and TRAIL was highly expressed in tumor tissues compared with adjacent normal samples (Fig. 1e, f). In addition, TRAIL was significantly increased in advanced stages (IIb-IV) compared with the early stages (I-Ila; Fig. 1f) and was negatively correlated with overall survival of patients with ESCC (Fig. 1g). Together, these data suggest that TRAIL accumulates in ESCC tumor sites and is negatively correlated with patient survival.

TRAIL is expressed by ESCC cells and knocking it down reduces ESCC cell migration in vitro

Initially, TRAIL was reported to be expressed by immune cells and that it defends against pathogens and self-antigens. However, TRAIL was found expressed by colon and lung cancers and a contributor to tumor 
progression in recent years[9]. Herein, TRAIL was found negatively correlated with patient prognosis, and immunohistochemistry data revealed that TRAIL was mainly expressed around the tumor cells. Thus, we hypothesized that ESCC cells may be the source of TRAIL. To determine whether ESCC cells express TRAIL, we examined TRAIL expression in seven human ESCC cell lines at different stages of differentiation (EC9706, TE7, EC1, KYSE150, KYSE70, TE-1, and EC109) at the mRNA and protein levels (Fig. 2a). TRAIL expression was found heterogeneous in ESCC cell lines, with KYSE150/KYSE70 cells exhibiting the highest expression levels (Fig. 2a).

To evaluate the possible biological function of TRAIL in ESCC, we silenced TRAIL in KYSE70 and KYSE150 cells using siRNAs. After verifying the silencing efficiency by RT-qPCR (Supplementary Fig. 1a), sphere formation, Transwell, and CCK8 assays were performed. The data showed that knocking down TRAIL significantly reduced the ability of cells to form spheroids, migrate, and proliferate (Supplementary Fig. 1d-f). Moreover, we analyzed stemness-related gene expression (Bmi1, Oct4, and KIf4) and found that TRAIL silencing decreased their gene expression levels (Supplementary Fig. 1g). These results suggest that TRAIL may enhance the stem properties of ESCC. To investigate this, we knocked down TRAIL using short hairpin RNAs (shRNAs); knockout efficiency was verified at both the mRNA and protein levels (Fig. 2b, c), after which sphere formation, Transwell, and CCK8 assays were performed. Similarly, knocking down TRAIL reduced ESCC cell migration, spheroid formation, and proliferation ability (Fig. 2df). Moreover, expression of the stemness-related genes Bmi1, Oct4, Cd44, and Klf4 significantly declined in TRAIL-knockdown KESE70 and KYSE150 cells; the change in Sox2 expression levels were not significant (Fig. 2g). Together, these results suggest that ESCC cells express TRAIL and that TRAIL may promote tumor stemness.

\section{Overexpression of TRAIL promotes ESCC cell migration, invasion, and proliferation in vitro}

We next stably overexpressed TRAIL in EC1 and TE1 cells, which showed the lowest TRAIL levels. After verifying the efficacy at both the mRNA and protein levels (Fig. 3a, b) we performed sphere formation, Transwell, and CCK8 assays. In contrast to TRAIL knockdown, overexpression significantly enhanced EC1/TE1 cell sphere formation (Fig. 3c), migration (Fig. 3d), and proliferation (Fig. 3e) abilities. As for the stemness-related genes, their expression was significantly increased in TRAIL overexpressed EC1 and TE1 cells compared with the control group (Fig. 3f). We further we used recombinant human TRAIL (rhTRAIL) to verify this phenomenon. Similar to the overexpression system, rh-TRAIL treatment significantly increased the expression of the stemness-related markers CD271, CXCR4, and Bmi1 (Supplementary Fig. 2a-c). Additionally, rh-TRAIL significantly promoted cellular migration and sphere formation (Supplementary Fig. 2d, e). Taken together, our data indicate that TRAIL facilitates the migration, invasion, proliferation, and stemness of ESCC cells.

\section{TRAIL promotes EMT of ESCC cells}


Previous studies have shown that tumor distant metastasis and migration mainly result from EMT[20] and that TRAIL regulates EMT in breast cancer[21]. Given that TRAIL was found expressed by ESCC cells, which promoted their migration and stemness, we investigated whether TRAIL promotes ESCC stemness and migration in an EMT-dependent manner. We examined the main markers associated with EMT and found that knocking out TRAIL significantly increased E-cadherin expression and markedly reduced $\mathrm{N}$ cadherin, vimentin, MMP2, and MMP9 levels (Fig. 4a, b). Of note, MMP3 was significantly elevated in shTRAIL-KYSE150 cells, but not in shTRAIL-KYSE70 cells (Fig. 4a, b). During the initial EMT step, cancer cells secrete MMPs to degrade collagen and fibrin[22]. MMP2/3/9 can degrade collagen and fibrin, which may explain why these two cell lines showed different MMP expression patterns. The TRAIL-induced modulation of EMT-related genes (E-cadherin, N-cadherin, and vimentin) was further validated at the protein level by western blotting (Fig. 4c). Similar results were obtained after using siRNA to knockdown TRAIL (Supplementary Fig. 3a-d).

On the other hand, when TRAIL was overexpressed in EC1 and TE1 cells, we observed the opposite trends at both the mRNA (Fig. 4d, e) and protein levels (Fig. 4e-g). Adding human recombinant TRAIL increased PD-L1, vimentin and $\mathrm{N}$-cadherin expression levels, reduced E-cadherin expression (Supplementary Fig. $3 e-h)$. Taken together, these results indicate that TRAIL promotes EMT of ESCC cells, which may underly the promotion of ESCC progression by TRAIL.

\section{TRAIL activates the ERK/STAT3 signaling pathway to induce PD-L1 expression in ESCC}

Next, we investigated the mechanism of TRAIL-induced EMT. Programmed death-ligand 1 (PD-L1), as an immunosuppressive molecule, is highly expressed on the surface of a variety of cancer cells[23]. Recently, it was reported that PD-L1 promotes EMT, and thus induces tumor progression in lung and breast cancer[24-26]. To investigate whether PD-L1 is involved in TRAIL-induced EMT, we first analyzed the expression of immunosuppressive molecules (CD274, TIM3, LAG3, CTLA4, CD38, CD101, MKI67, IC0S, EOMES, CD44, CD28, KLRG1, CD5, TIGIT, and CD47) in the TRAIL high and low groups in TCGA database[27]. We found that PD-L1 (CD274) and CTLA4 show significantly higher expression in the TRAIL $^{\text {high }}$ group than in the TRAIL ${ }^{\text {low }}$ group (Fig. 5a); however, PD-L1 (CD274) was predominantly expressed by tumors[28] while CTLA4 and LAG3 were mostly expressed by T cells in the tumor microenvironment[29]. Notably, a markedly positive correlation was observed between PD-L1 (CD274) and TRAIL (TNFSF10) in ESCC tissues (Fig. 5b). To confirm this, we performed immunohistochemistry of ESCC samples collected from the patients. Similar expression results were obtained, and a significant correlation between PD-L1 and TRAIL was observed (Fig. 5c, d). Furthermore, we examined TRAILknockout and overexpressed cells and found that PD-L1 expression was lower in the knockdown group and higher in the overexpression group than in control (Fig. 5e). Thus, these results suggest that TRAIL regulates PD-L1 expression in ESCC.

We then assessed the mechanism underlying TRAIL regulation of PD-L1 expression. As a key molecule in tumor immune escape, the main mechanisms involved in PD-L1 regulation have been reported. We first 
examined the KEGG enrichment analysis in TCGA and found that only the MAPK/STAT3 signaling pathway was significantly up-regulated in tissues with high TRAIL expression (Fig. 5f). To reveal whether TRAIL-induced PD-L1 expression depends on MAPK/STAT3, we performed western blotting of TRAILknockout and overexpressed ESCC cells. Knocking down TRAIL in KYSE150 cells markedly reduced p-ERK and p-STAT3 expression, while overexpression in EC1 cells significantly increased p-ERK and p-STAT3 levels (Fig. 5g). Furthermore, treatment of EC1 cells with ERK or STAT3 inhibitors reversed the TRAIL overexpression-induced increase in PD-L1 levels (Fig. 5h, i).

When PD-L1 antibody was added to TRAIL-overexpression EC1 cells, only N-cadherin was significantly downregulated, while E-cadherin and vimentin showed no difference in mRNA levels (Supplementary Fig. 4a). However, after silencing PD-L1 in TRAIL-overexpression EC1 cells, both N-cadherin and vimentin were markedly downregulated, while E-cadherin expression did not change (Supplementary Fig. 4b). The aggressive and proliferation abilities of tumor cells were also inhibited (Supplementary Fig. 4c, d). These

findings indicate that cytoplasmic PD-L1 promotes EMT, whereas PD-L1 expressed on the cell membrane cannot promote cell metastasis and invasion. Altogether, the results demonstrate that TRAIL-induced EMT is dependent on ERK/STAT3-activated PD-L1 expression in ESCC.

\section{TRAIL activates the ERK/STAT3 pathway, inducing PD-L1 and promoting EMT of ESCC cells in vivo}

We next examined whether the TRAIL-induced promotion of EMT through ERK/STAT3-activated PD-L1 expression in vitro occurs in vivo using mouse models. Knocking down TRAIL significantly delayed the tumor growth of KYSE150 cells (Fig. 6a, b). Moreover RT-qPCR and western blotting showed a significant increase in E-cadherin expression and a marked decrease in the expression of $\mathrm{N}$-cadherin, vimentin, $\mathrm{PD}$ L1, MMP2, MMP3, MMP9, and stemness-related genes (Sox2, Bmi1, Oct4, and Cd44) in shTRAILKYSE150 cells compared with control (Fig. 6c, d). Meanwhile, immunohistochemistry data showed a significant positive correlation between TRAIL and PD-L1 (Fig. 6e, f). In contrast, overexpression of TRAIL in EC1 cells significantly accelerated tumor growth (Supplementary Fig. 5a, b) and promoted EMT- and stemness-related gene expression at the mRNA and protein levels (Supplementary Fig. 5c, d). Moreover, overexpression of TRAIL upregulated p-ERK/p-STAT3/PD-L1 levels (Supplementary Fig. 5d). The correlation between TRAIL and PD-L1 was also demonstrated (Supplementary Fig. 5e-g). Taken together, these data indicate that TRAIL promotes EMT-induced cell metastasis through ERK/STAT3 signaling pathway activation and inducing PD-L1 expression in ESCC in vivo.

\section{Discussion}

In this study, we identified an ERK/STAT3-dependent mechanism whereby TRAIL induces PD-L1 expression, which in turn promotes EMT and tumor progression in ESCC. The role of TRAIL in tumors is still poorly understood. Although TRAIL was initially considered as the important Inflammatory cytokines of immune cells, many clinical trials with recombinant human TRAIL or TRAIL receptor agonists have failed. TRAIL is also expressed by cancer cells and functions as a tumor promoter. However, the role of 
TRAIL in ESCC is poorly defined. Herein, we found that TRAIL accumulated in ESCC tumor tissues and was negatively correlated with clinical parameters as well as patient overall survival. Furthermore, we found that ESCC cells express TRAIL, which induced EMT and stemness through the ERK/STAT3/PD-L1 pathway. These results highlight the unique features of TRAIL in ESCC.

To date, the role of TRAIL in cancer remains controversial, as its function varies depending on cell type. Initially, TRAIL, as a secondary transmembrane protein, was reported to be mainly expressed on the surface of immune cells and involved in the immune response. However, the diversity of TRAIL functions was gradually revealed. Singh et al.[30] reported that the cytotoxic effects of T cells predominantly rely on the expression of TRAIL on their surface. Several studies have shown that TRAIL promotes apoptosis in cancer cells[31-33]. These results indicate that TRAIL plays an antitumor role in the tumor microenvironment. Interestingly, a few studies reported that TRAIL can promote tumor invasion and metastasis in tumors with KRAS mutations[9, 34, 35], and that TRAIL promotes NF-KB-dependent tumor cell migration and invasion without influencing proliferation[36]. TRAIL was also shown to promote the immunosuppressive cancer microenvironment by inducing Treg proliferation[37, 38]. In addition, TRAIL can induce pro-inflammatory cytokines to polarize myeloid cells toward myeloid-derived suppressor cells and complete differentiation of the M2 macrophage phenotype[9, 39]. However, the main source of TRAIL and its cytological function in esophageal cancer is still not well understood. Herein, we found that TRAIL was mainly produced by tumor cells and promoted their metastasis and invasion in ESCC. For the first time, we revealed the biological role of TRAIL in ESCC, expanding the diversity of TRAIL functions. Both tumor cells and immune cells can express TRAIL, but they have different functions; however, we did not perform further studies on this functional difference. Based on the reported studies, these discrepant functions may be due to: (1) the different states of the tumor microenvironment; (2) structural differences between these two different sources of TRAIL; and (3) different concentrations of TRAIL in the tumor microenvironment. Indeed, it has been reported that low doses of TRAIL promote tumor cell invasion and metastasis by activating p-STAT3 in non-small cell lung cancer $[4,40]$ and that TRAIL induces a significant amount of apoptosis in a human T-cell line in a dose-dependent manner[41].

EMT is a key process in tumor invasion and metastasis. Various factors contribute to EMT, including low oxygen, low pH acidic environment, stromal cells, and tumor cells, as well as the secretion of various inflammatory factors and cytokines by immune cells[12, 42]. In recent years, immune checkpoint inhibitors have also been reported to be associated with EMT, especially PD-L1. Using pancreatic cancer samples, it was reported that PD-L1 promotes EMT and carcinogenesis[15]. Additionally, the role of PD-L1 in regulating EMT has also been reported in lung cancer[14, 43] and esophageal cancer[13]. Consistent with previous studies, we demonstrated that TRAIL-mediated EMT in ESCC is dependent on PD-L1 expression in the cytoplasm.

Some studies have reported that TRAIL and PD-L1 expression is consistent in lung and Gastric Cancer[17, $44,45]$, but there are no relevant studies exploring their mechanisms. Mitogen activated protein kinases (MAPKs) are a class of serine/threonine protein kinases. The MAPK signal transduction pathway is present in most cells and plays a crucial role in transducing extracellular signals to the intracellular 
nucleus, causing biological responses such as cell proliferation, differentiation, and transformation[46]. Three parallel MAPK signaling pathways have been identified in cells: (1) the ERK pathway, which is considered the classical MAPK signaling pathway; (2) the JNK/SAPK pathway; and (3) the p38MAPK pathway[47]. STAT3, signal transducer and activator of transcription 3, plays an important role in the progression of multiple tumors. It has also been reported that the upregulation of MAPK can specifically promote the phosphorylation of a serine (Ser) at the C-terminus of STAT3, which greatly enhances the transcriptional activity of STAT3[48]. It has also been reported that ERK1/2 and STAT3 increase the resistance of tumor cells to TRAIL-promoted apoptosis[49]. In our study, we found for the first time that TRAIL can promote PD-L1 expression through the ERK/STAT3 signaling pathway and promote EMT, as evidenced by TCGA database and western blotting. Hence, we speculate that STAT3/ERK inhibitors can be combined with TRAIL recombinant proteins to treat tumors, which warrants further studies.

In conclusion, although TRAIL is known to be expressed by immune cells, this is the first report to reveal that the source of TRAIL expression in ESCC is ESCC tumor cells. Furthermore, we investigated the correlation between TRAIL and PD-L1 and found that TRAIL regulates PD-L1 expression through the STAT3/ERK signaling pathway, further inducing EMT and tumor progression in ESCC (Fig. 6g).

\section{Conclusions}

In this study, we found that TRAIL was a poor predictor in patients with ESCC. Further research found that TRAIL was expressed by ESCC cells and can induce EMT by upregulating PD-L1 expression, meanwhile the process was activated through the ERK/STAT3 signaling pathway. Thus, TRAIL has the potential to act as a key molecule to target EMT and inhibit ESCC progression in the future.

\section{Abbreviations}

TRAIL

Tumor necrosis factor-associated apoptosis-inducing ligand; TNFSF:tumor necrosis factor superfamily; OPG:osteoprotegerin; ESCC:esophageal squamous cell carcinoma; EMT:epithelial-mesenchymal transition; PD-L1:Programmed death-ligand 1; MMPs:Matrix Metalloproteinases; siRNA:Small interfering RNA; rh-TRAIL:recombinant human TRAIL; STAT3:Signal transducer and activator of transcription 3; MAPKs:Mitogen activated protein kinases; shRNAs:short hairpin RNAs; PBS:Phosphate buffered saline; RT-qPCR:Quantitative real-time polymerase chain reaction; SD:Standard deviation.

\section{Declarations}

\section{Acknowledgements}

Studies involving clinical ESCC samples were reviewed and approved by the First Affiliated Hospital of Zhengzhou University Research Ethics Committee.

\section{Author Contributions}


H. Zhang, G. Qin, and J. Liu were responsible for the overall experimental design; H. Zhang and H. Yang performed most experiments and data analysis; $\mathrm{P}$. Wu and $\mathrm{H}$. Hu assisted with animal experiments and western blotting; L. Yang and S. Liu provided guidance; H. Zhang and J. Liu wrote the manuscript; Y. Zhang revised the manuscript and supervised the study.

\section{Funding}

This work was supported by the National Key Research and Development (2018YFC1313400), the National Nature Science Foundation of China (U1804281, 91942314) and the National Science Fund for Distinguished Young Scholars (82001659).

\section{Availability of data and materials}

The data sets used and analyzed during the current study are available from the corresponding author on reasonable request.

\section{Ethics approval and consent to participate}

$\mathrm{N} / \mathrm{A}$

\section{Consent for publication}

$\mathrm{N} / \mathrm{A}$

\section{Competing interests}

The authors state no conflict of interest.

\section{Author details}

${ }^{1}$ Biotherapy Center \& Cancer Center, the First Affiliated Hospital, Zhengzhou University, Zhengzhou 450052, China. ${ }^{2}$ State Key Laboratory of Esophageal Cancer Prevention \& Treatment, Zhengzhou 450052 , Henan, China. ${ }^{3}$ School of Life Sciences, Zhengzhou University, Zhengzhou 450052, China. ${ }^{4}$ Henan Key Laboratory for Tumor Immunology and Biotherapy, Zhengzhou 450052, China

\section{References}

1. Emery JG, McDonnell P, Burke MB, Deen KC, Lyn S, Silverman C, et al. Osteoprotegerin is a receptor for the cytotoxic ligand TRAIL. J Biol Chem. 1998;273(23):14363-7.

2. Pan G, O'Rourke K, Chinnaiyan AM, Gentz R, Ebner R, Ni J, et al. The receptor for the cytotoxic ligand TRAIL. Science. 1997;276(5309):111-3.

3. Schneider P, Thome M, Burns K, Bodmer JL, Hofmann K, Kataoka T, et al. TRAIL receptors 1 (DR4) and 2 (DR5) signal FADD-dependent apoptosis and activate NF-kappaB. Immunity. 1997;7(6):831-6. 
4. von Karstedt S, Montinaro A, Walczak H. Exploring the TRAlLs less travelled: TRAIL in cancer biology and therapy. Nat Rev Cancer. 2017;17(6):352-66.

5. Cardoso Alves L, Berger MD, Koutsandreas T, Kirschke N, Lauer C, Sporri R, et al. Non-apoptotic TRAIL function modulates NK cell activity during viral infection. EMBO Rep. 2020;21(1):e48789.

6. Chyuan IT, Hsu PN. TRAIL regulates T cell activation and suppresses inflammation in autoimmune diseases. Cell Mol Immunol. 2020.

7. Qiao X, Wang X, Shang Y, Li Y, Chen SZ. Azithromycin enhances anticancer activity of TRAIL by inhibiting autophagy and up-regulating the protein levels of DR4/5 in colon cancer cells in vitro and in vivo. Cancer Commun (Lond). 2018;38(1):43.

8. Yang A, Wilson NS, Ashkenazi A. Proapoptotic DR4 and DR5 signaling in cancer cells: toward clinical translation. Curr Opin Cell Biol. 2010;22(6):837-44.

9. Hartwig T, Montinaro A, von Karstedt S, Sevko A, Surinova S, Chakravarthy A, et al. The TRAILInduced Cancer Secretome Promotes a Tumor-Supportive Immune Microenvironment via CCR2. Mol Cell. 2017;65(4):730-42 e5.

10. Lambert AW, Pattabiraman DR, Weinberg RA. Emerging Biological Principles of Metastasis. Cell. 2017;168(4):670-91.

11. Pastushenko I, Blanpain C. EMT Transition States during Tumor Progression and Metastasis. Trends Cell Biol. 2019;29(3):212-26.

12. Jiang Y, Zhan H. Communication between EMT and PD-L1 signaling: New insights into tumor immune evasion. Cancer letters. 2020;468:72-81.

13. Chen L, Xiong Y, Li J, Zheng X, Zhou Q, Turner A, et al. PD-L1 Expression Promotes Epithelial to Mesenchymal Transition in Human Esophageal Cancer. Cell Physiol Biochem. 2017;42(6):2267-80.

14. Hong W, Xue M, Jiang J, Zhang Y, Gao X. Circular RNA circ-CPA4/ let-7 miRNA/PD-L1 axis regulates cell growth, stemness, drug resistance and immune evasion in non-small cell lung cancer (NSCLC). J Exp Clin Cancer Res. 2020;39(1):149.

15. Zhang H, Zhu C, He Z, Chen S, Li L, Sun C. LncRNA PSMB8-AS1 contributes to pancreatic cancer progression via modulating miR-382-3p/STAT1/PD-L1 axis. J Exp Clin Cancer Res. 2020;39(1):179.

16. Reguera-Nunez E, Xu P, Chow A, Man S, Hilberg F, Kerbel RS. Therapeutic impact of Nintedanib with paclitaxel and/or a PD-L1 antibody in preclinical models of orthotopic primary or metastatic triple negative breast cancer. J Exp Clin Cancer Res. 2019;38(1):16.

17. Lv J, Guo T, Qu X, Che X, Li C, Wang S, et al. PD-L1 Under Regulation of miR-429 Influences the Sensitivity of Gastric Cancer Cells to TRAIL by Binding of EGFR. Front Oncol. 2020;10:1067.

18. Smyth EC, Lagergren J, Fitzgerald RC, Lordick F, Shah MA, Lagergren P, et al. Oesophageal cancer. Nat Rev Dis Primers. 2017;3:17048.

19. Allemani C, Matsuda T, Di Carlo V, Harewood R, Matz M, Niksic M, et al. Global surveillance of trends in cancer survival 2000-14 (CONCORD-3): analysis of individual records for 37513025 patients 
diagnosed with one of 18 cancers from 322 population-based registries in 71 countries. Lancet. 2018;391(10125):1023-75.

20. Wei Q, Qian Y, Yu J, Wong CC. Metabolic rewiring in the promotion of cancer metastasis: mechanisms and therapeutic implications. Oncogene. 2020;39(39):6139-56.

21. Srivastava RK, Kurzrock R, Shankar S. MS-275 sensitizes TRAIL-resistant breast cancer cells, inhibits angiogenesis and metastasis, and reverses epithelial-mesenchymal transition in vivo. Mol Cancer Ther. 2010;9(12):3254-66.

22. Torzilli PA, Bourne JW, Cigler T, Vincent CT. A new paradigm for mechanobiological mechanisms in tumor metastasis. Semin Cancer Biol. 2012;22(5-6):385-95.

23. Lei Q, Wang D, Sun K, Wang L, Zhang Y. Resistance Mechanisms of Anti-PD1/PDL1 Therapy in Solid Tumors. Frontiers in Cell and Developmental Biology. 2020;8.

24. De Matteis S, Canale M, Verlicchi A, Bronte G, Delmonte A, Crino L, et al. Advances in Molecular Mechanisms and Immunotherapy Involving the Immune Cell-Promoted Epithelial-to-Mesenchymal Transition in Lung Cancer. J Oncol. 2019;2019:7475364.

25. Noman MZ, Janji B, Abdou A, Hasmim M, Terry S, Tan TZ, et al. The immune checkpoint ligand PDL1 is upregulated in EMT-activated human breast cancer cells by a mechanism involving ZEB-1 and miR-200. Oncoimmunology. 2017;6(1):e1263412.

26. Wang $\mathrm{H}$, Xu C, Kong X, Li X, Kong X, Wang $Y$, et al. Trail resistance induces epithelial-mesenchymal transition and enhances invasiveness by suppressing PTEN via miR-221 in breast cancer. PLoS One. 2014;9(6):e99067.

27. Waite JC, Wang B, Haber L, Hermann A, Ullman E, Ye X, et al. Tumor-targeted CD28 bispecific antibodies enhance the antitumor efficacy of PD-1 immunotherapy. Science translational medicine. 2020;12(549).

28. Baba $Y$, Nomoto D, Okadome $K$, Ishimoto T, Iwatsuki M, Miyamoto $Y$, et al. Tumor immune microenvironment and immune checkpoint inhibitors in esophageal squamous cell carcinoma. Cancer Sci. 2020;111(9):3132-41.

29. Zhou G, Sprengers D, Boor PPC, Doukas M, Schutz H, Mancham S, et al. Antibodies Against Immune Checkpoint Molecules Restore Functions of Tumor-Infiltrating T Cells in Hepatocellular Carcinomas. Gastroenterology. 2017;153(4):1107-19 e10.

30. Singh N, Lee YG, Shestova O, Ravikumar P, Hayer KE, Hong SJ, et al. Impaired Death Receptor Signaling in Leukemia Causes Antigen-Independent Resistance by Inducing CAR T-cell Dysfunction. Cancer Discov. 2020;10(4):552-67.

31. Zhang X, Zhang X, Hu S, Zheng M, Zhang J, Zhao J, et al. Identification of miRNA-7 by genome-wide analysis as a critical sensitizer for TRAIL-induced apoptosis in glioblastoma cells. Nucleic Acids Res. 2017;45(10):5930-44.

32. Kim BR, Park SH, Jeong YA, Na YJ, Kim JL, Jo MJ, et al. RUNX3 enhances TRAIL-induced apoptosis by upregulating DR5 in colorectal cancer. Oncogene. 2019;38(20):3903-18. 
33. Xiao R, An Y, Ye W, Derakhshan A, Cheng H, Yang X, et al. Dual Antagonist of clAP/XIAP ASTX660 Sensitizes HPV(-) and HPV(+) Head and Neck Cancers to TNFalpha, TRAIL, and Radiation Therapy. Clin Cancer Res. 2019;25(21):6463-74.

34. von Karstedt S, Conti A, Nobis M, Montinaro A, Hartwig T, Lemke J, et al. Cancer cell-autonomous TRAIL-R signaling promotes KRAS-driven cancer progression, invasion, and metastasis. Cancer cell. 2015;27(4):561-73.

35. Pal S, Amin PJ, Sainis KB, Shankar BS. Potential Role of TRAIL in Metastasis of Mutant KRAS Expressing Lung Adenocarcinoma. Cancer Microenviron. 2016;9(2-3):77-84.

36. Ishimura N, Isomoto H, Bronk SF, Gores GJ. Trail induces cell migration and invasion in apoptosisresistant cholangiocarcinoma cells. Am J Physiol Gastrointest Liver Physiol. 2006;290(1):G129-36.

37. Ikeda T, Hirata S, Fukushima S, Matsunaga $Y$, Ito T, Uchino M, et al. Dual effects of TRAIL in suppression of autoimmunity: the inhibition of Th1 cells and the promotion of regulatory $T$ cells. $J$ Immunol. 2010;185(9):5259-67.

38. Pillai MR, Collison LW, Wang X, Finkelstein D, Rehg JE, Boyd K, et al. The plasticity of regulatory T cell function. J Immunol. 2011;187(10):4987-97.

39. Trauzold A, Siegmund D, Schniewind B, Sipos B, Egberts J, Zorenkov D, et al. TRAIL promotes metastasis of human pancreatic ductal adenocarcinoma. Oncogene. 2006;25(56):7434-9.

40. Azijli K, Yuvaraj S, Peppelenbosch MP, Wurdinger T, Dekker H, Joore J, et al. Kinome profiling of noncanonical TRAIL signaling reveals RIP1-Src-STAT3-dependent invasion in resistant non-small cell lung cancer cells. J Cell Sci. 2012;125(Pt 19):4651-61.

41. Chyuan IT, Tsai HF, Liao HJ, Wu CS, Hsu PN. An apoptosis-independent role of TRAIL in suppressing joint inflammation and inhibiting T-cell activation in inflammatory arthritis. Cell Mol Immunol. 2018;15(9):846-57.

42. Ma HY, Liu XZ, Liang CM. Inflammatory microenvironment contributes to epithelial-mesenchymal transition in gastric cancer. World J Gastroenterol. 2016;22(29):6619-28.

43. Shen M, Xu Z, Xu W, Jiang K, Zhang F, Ding Q, et al. Inhibition of ATM reverses EMT and decreases metastatic potential of cisplatin-resistant lung cancer cells through JAK/STAT3/PD-L1 pathway. $J$ Exp Clin Cancer Res. 2019;38(1):149.

44. Asgarova A, Asgarov K, Godet Y, Peixoto P, Nadaradjane A, Boyer-Guittaut M, et al. PD-L1 expression is regulated by both DNA methylation and NF-kB during EMT signaling in non-small cell lung carcinoma. Oncoimmunology. 2018;7(5):e1423170.

45. Hendriks D, He Y, Koopmans I, Wiersma VR, van Ginkel RJ, Samplonius DF, et al. Programmed Death Ligand 1 (PD-L1)-targeted TRAIL combines PD-L1-mediated checkpoint inhibition with TRAILmediated apoptosis induction. Oncoimmunology. 2016;5(8):e1202390.

46. Rezatabar S, Karimian A, Rameshknia V, Parsian H, Majidinia M, Kopi TA, et al. RAS/MAPK signaling functions in oxidative stress, DNA damage response and cancer progression. J Cell Physiol. 2019.

47. Fang JY, Richardson BC. The MAPK signalling pathways and colorectal cancer. Lancet Oncol. 2005;6(5):322-7. 
48. Wang $Y$, Shen $Y$, Wang S, Shen Q, Zhou X. The role of STAT3 in leading the crosstalk between human cancers and the immune system. Cancer Lett. 2018;415:117-28.

49. Amarante-Mendes GP, Griffith TS. Therapeutic applications of TRAIL receptor agonists in cancer and beyond. Pharmacol Ther. 2015;155:117-31.

Figures

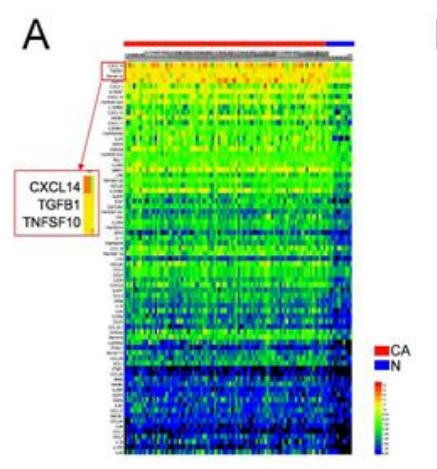

C
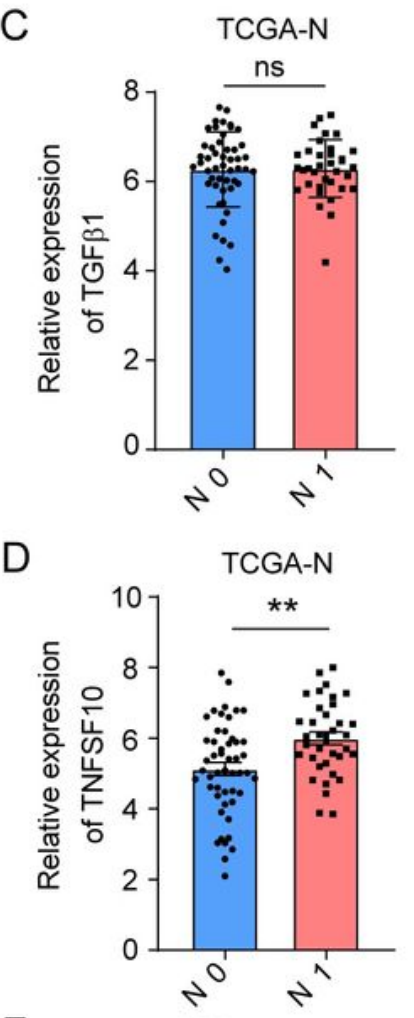

$\mathrm{F}$



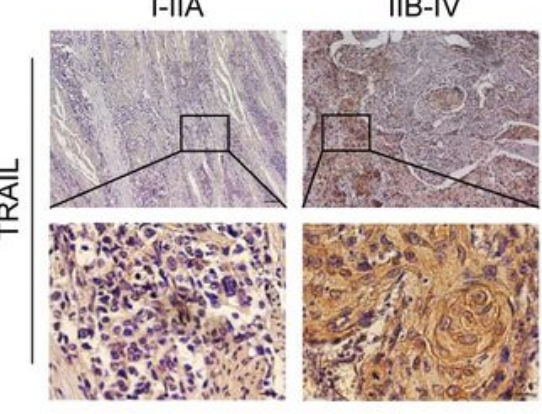

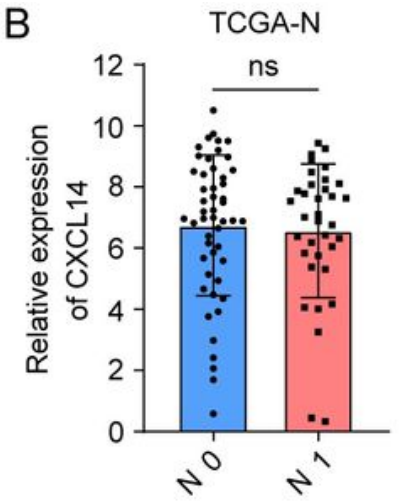
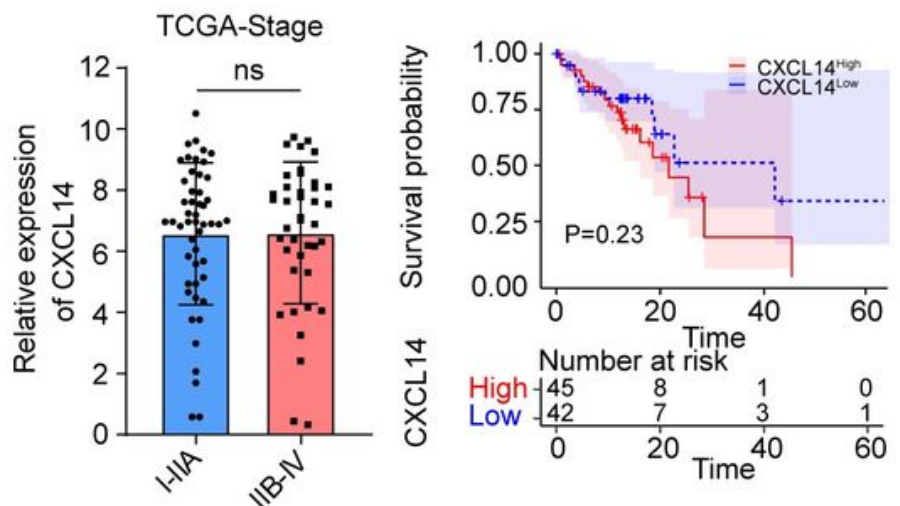

TCGA-Stage
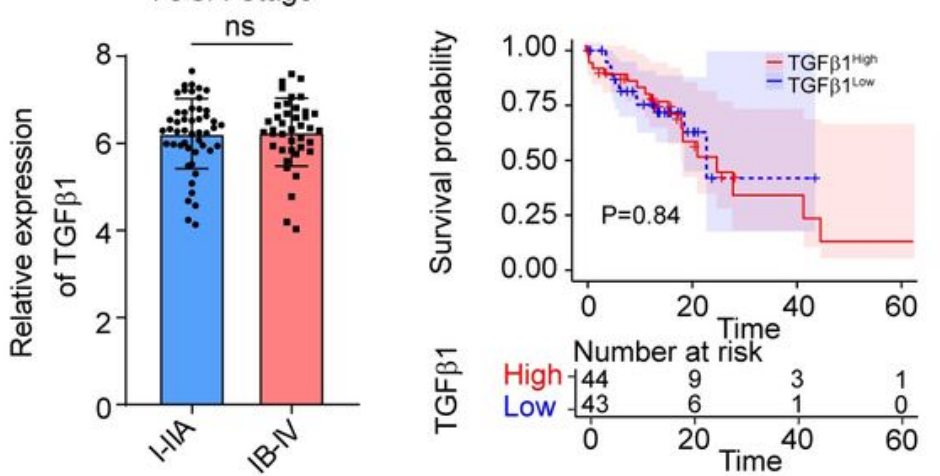

离
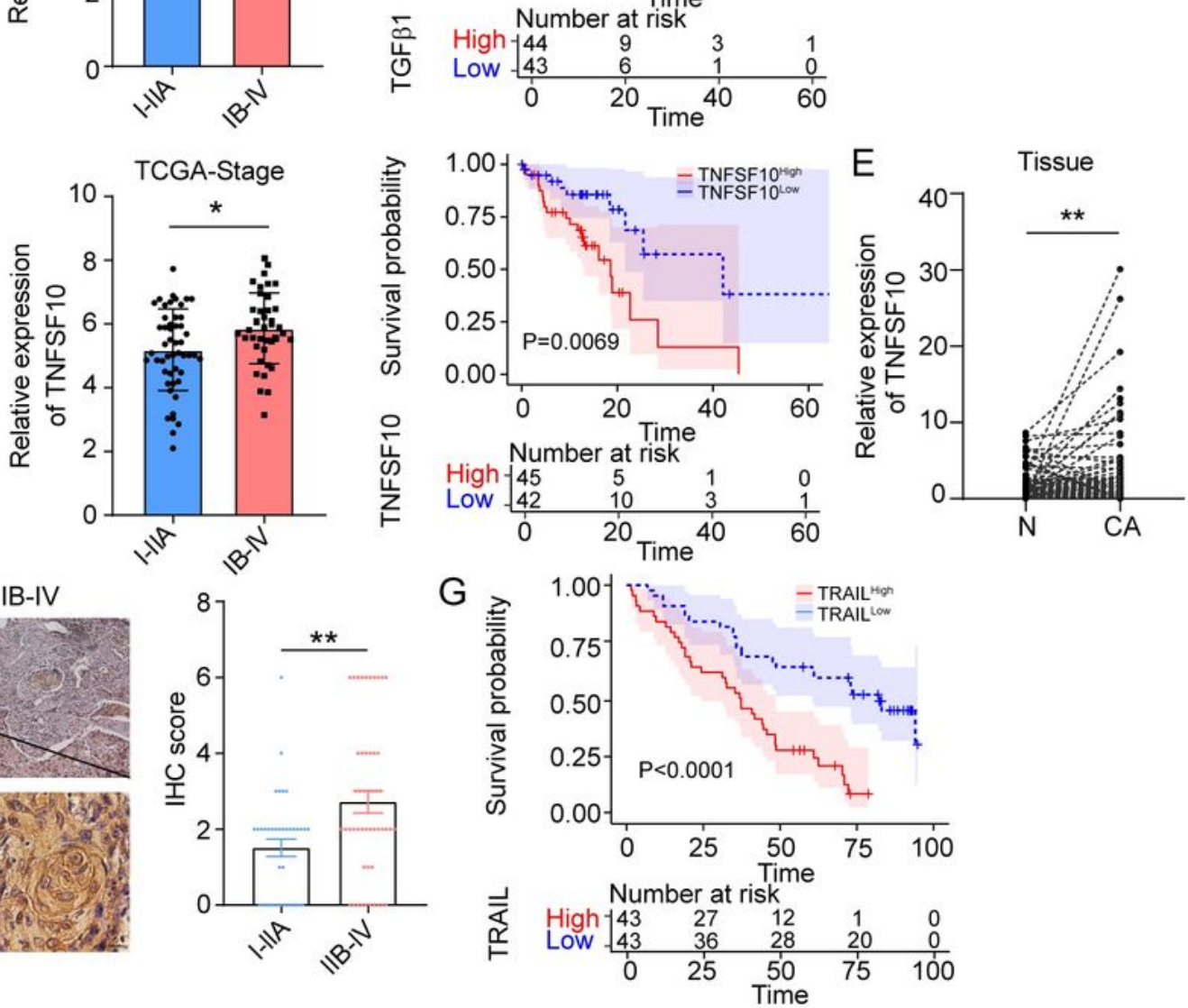


\section{Figure 1}

High expression of TRAIL in ECSS is positively correlated with lymph node metastasis, stage, and clinical prognosis of patients. (a) Comparison of differential genes in ESCC tissues $(n=93)$ and normal esophageal tissues $(n=11)$ in TCGA database. $(b-d)$ correlation of TGFB1, CXCL14, and TNFSF10 expression with metastasis, staging and clinical prognosis in patients in TCGA database. (e) The expression of TRAIL in cancer tissues is higher than in adjacent normal tissues at the mRNA level $(n=$ 83). (f) Immunohistochemical results showed higher expression of TRAIL in advanced tumors than in early tumor tissues. (g) High TRAlL expression is negatively associated with clinical survival. 
A
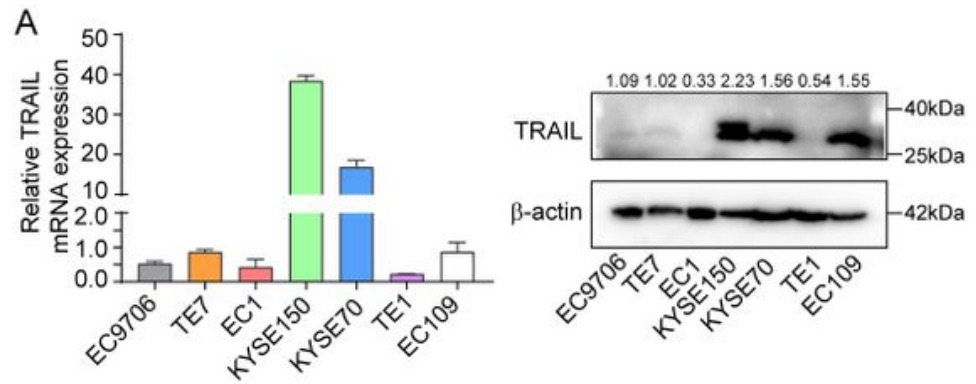

B

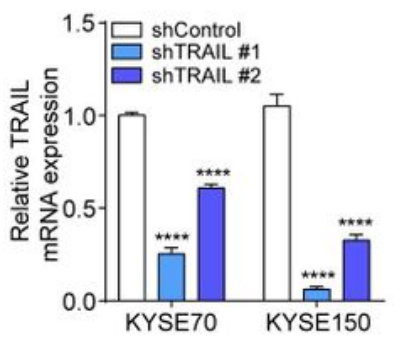

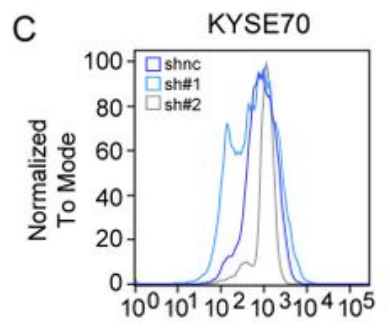
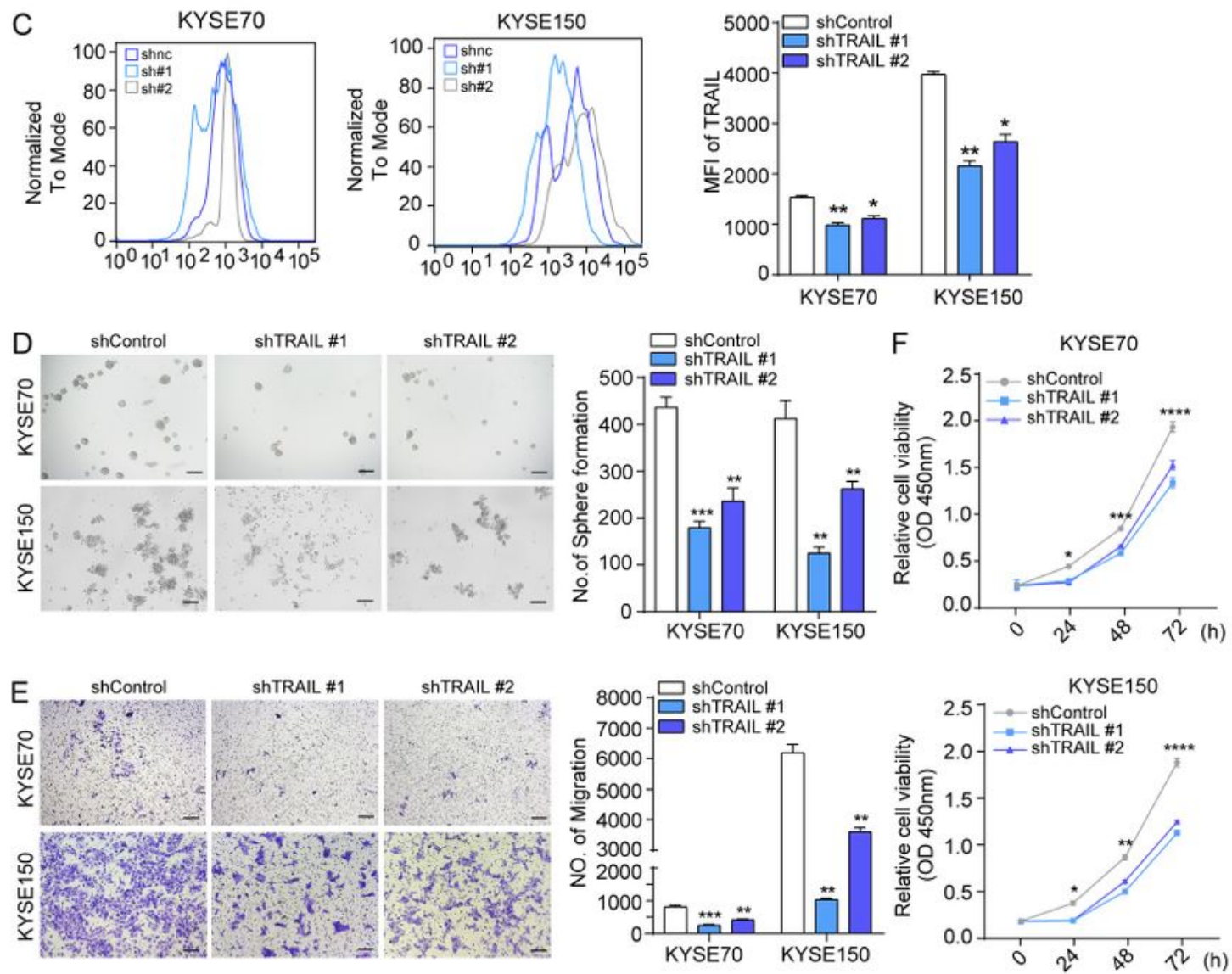

G
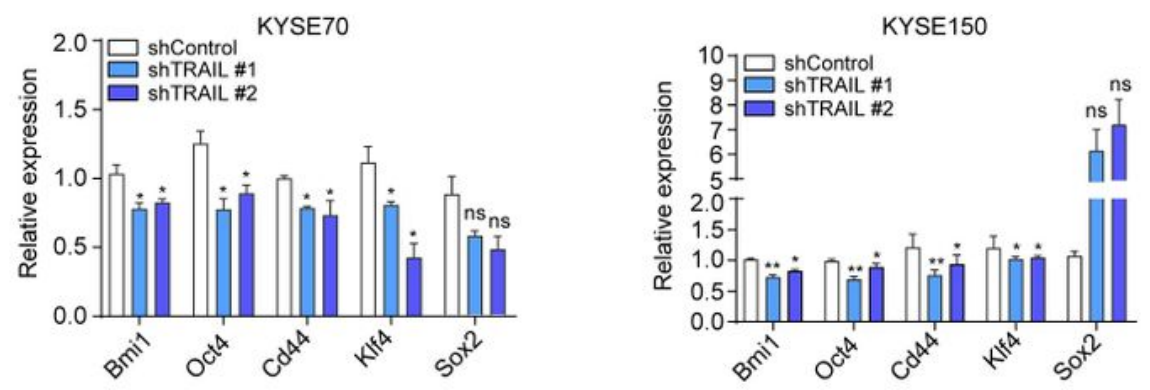

\section{Figure 2}

Knockdown of TRAIL inhibits ESCC cell migration, invasion, and proliferation in vitro. (a) TRAIL is differentially expressed in different ESCC samples as evidenced by RT-qPCR and western blotting. (b, c) The TRAIL knockdown effects in KYSE70 and KYSE150 cells were confirmed by RT-qPCR and flow cytometry. GAPDH was used as an internal loading control. (d) The effect of TRAIL on sphere formation of KYSE70 (top) and KYSE150 (bottom) cells. Representative images and quantitative analysis are 
shown in the left and right panels, respectively. (e) Transwell assay of TRAIL knockdown and control KYSE70 and KYSE150 cells. Representative images and quantitative analysis are shown in the left and right panels, respectively. (f) Cell proliferation determined by the CCK-8 assay with/without TRAIL knockdown in KYSE70 (top) or KYSE150 (bottom) cells. (g) RT-qPCR of cell stemness markers in KYSE70 (left) or KYSE150 (right) cells. NC, negative control. The data are presented as the mean \pm SEM of three independent experiments. ${ }^{* * *} \mathrm{P}<0.0001,{ }^{*} * \mathrm{P}<0.001,{ }^{*} \mathrm{P}<0.01$, ${ }^{*} \mathrm{P}<0.05$ (paired $\mathrm{t}$-test).

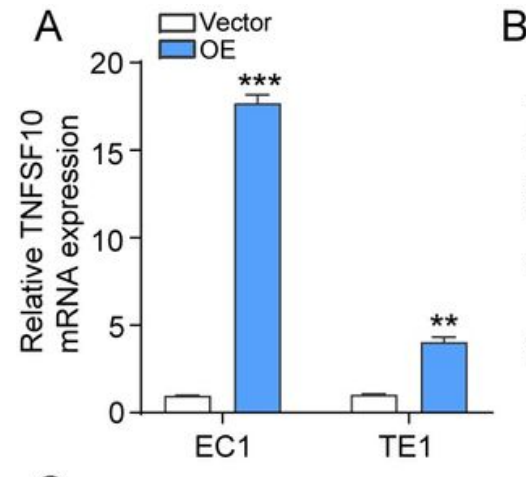

C

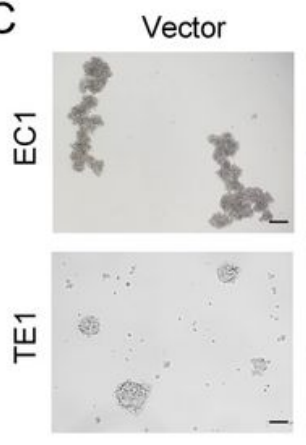

D

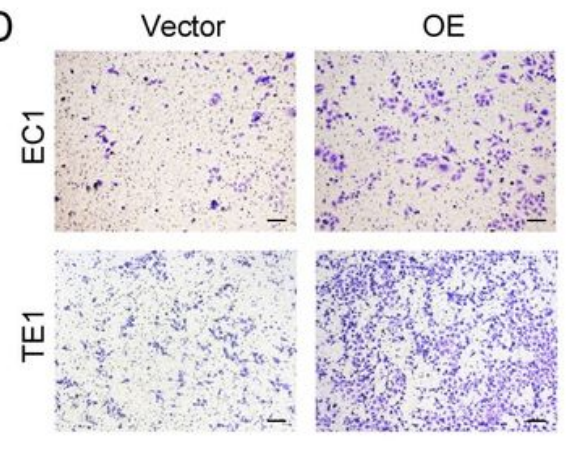

$\mathrm{F}$

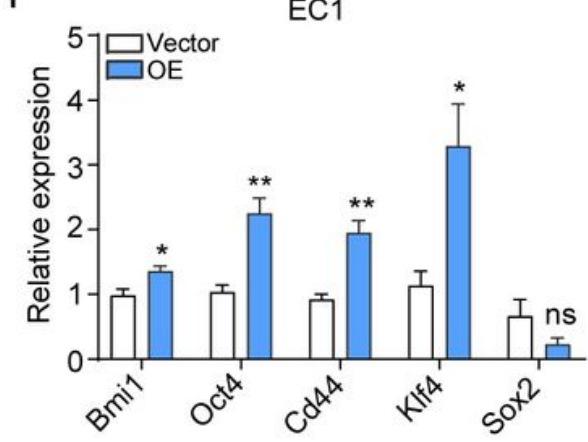

$\mathrm{OE}$

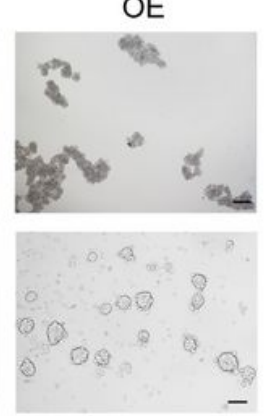

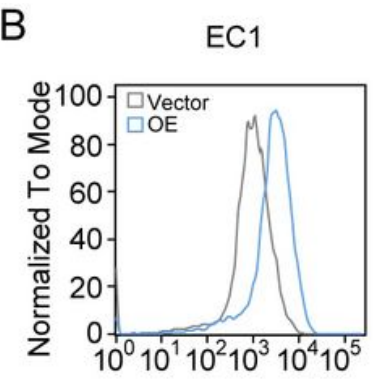
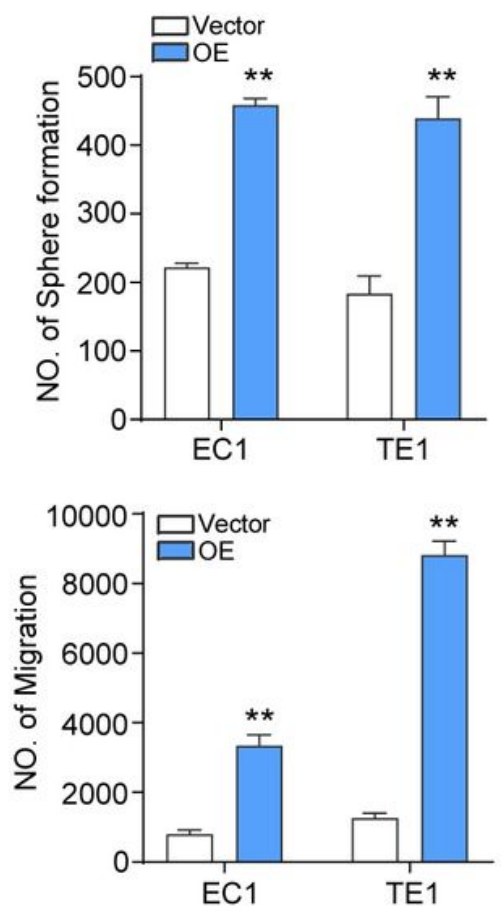

TE1

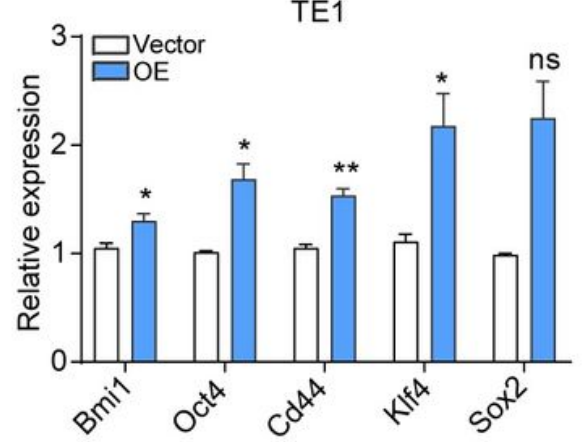

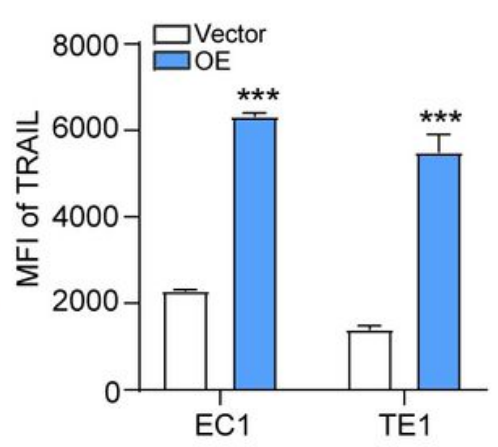

E
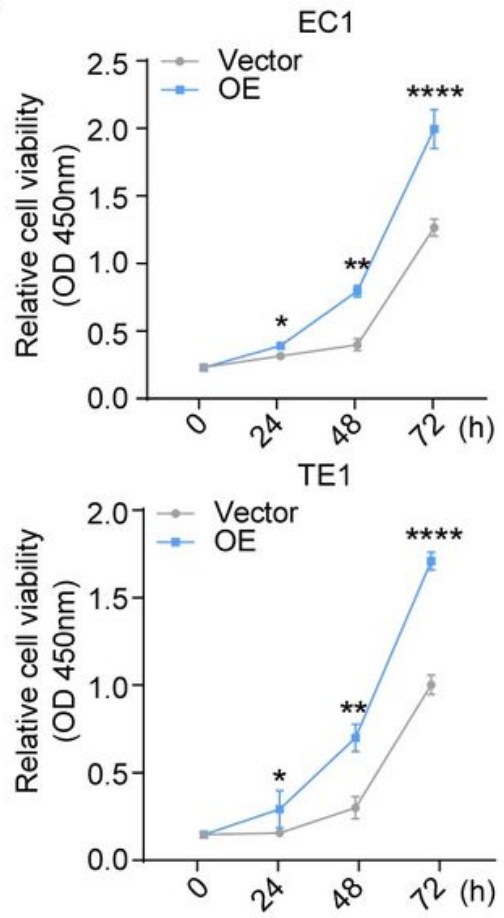

Figure 3 
Overexpression of TRAIL promotes ESCC cell migration, invasion, and proliferation. $(a, b)$ TRAIL overexpression effects in EC1 and TE1 cells were confirmed by RT-qPCR (left) and flow cytometry (right). (c, d) Sphere formation and transwell assays of TRAIL overexpression and control EC1 and TE1 cells. Representative images and quantitative analysis are shown in the left and right panels, respectively. (e) CCK-8 assay to determine cell proliferative capacity of EC1 (top) and TE1 (bottom) cells. (f) RT-qPCR of cell stemness-related genes, using GAPDH as a reference gene. NC, negative control. The data are presented as the mean $\pm S D$ of three independent experiments. ${ }^{\star \star \star *} P<0.0001$, ${ }^{\star \star \star} P<0.001,{ }^{\star *} P<0.01$, $\star \mathrm{P}<0.05$ (paired t-test). 

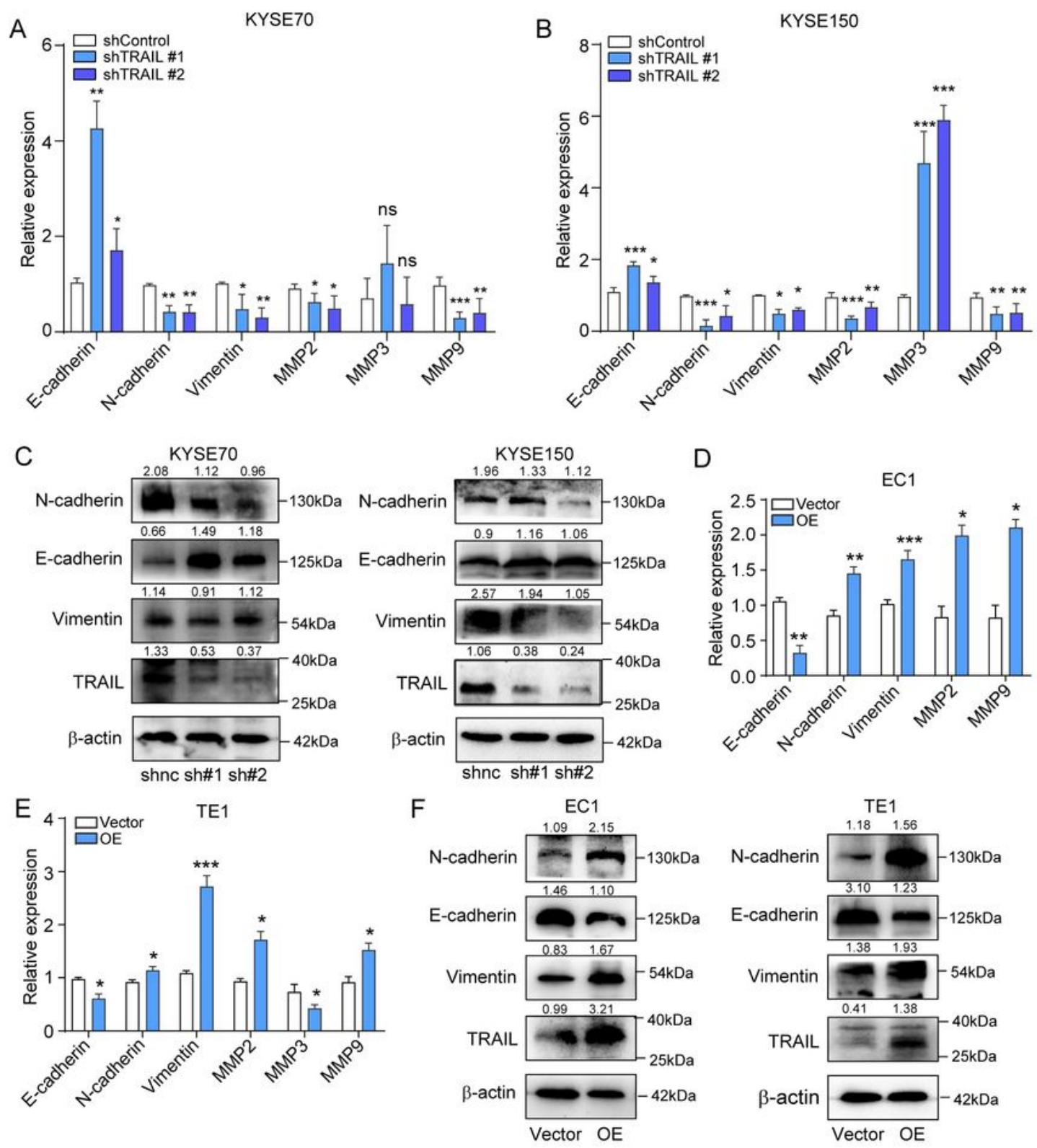

G

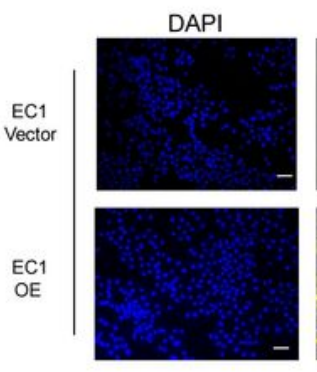

E-cadherin
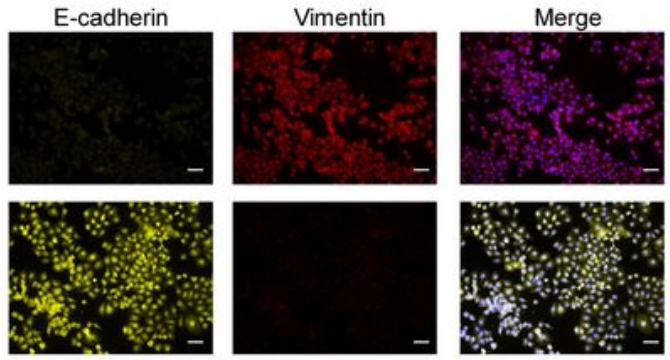

Figure 4

TRAIL facilitates epithelial-mesenchymal transition (EMT) by regulating the expression of EMT-related proteins. $(a, b)$ RT-qPCR of EMT-related genes in TRAIL-knockout KYSE70 and KYSE150 cells. (c) Western blotting of EMT-related proteins in TRAIL-knockout KYSE70 or KYSE150 cells and the respective controls. $(\mathrm{d}-\mathrm{f})$ mRNA and protein levels of EMT-related genes in TRAIL-overexpressing EC1 and TE1 cells. (g) Immunohistochemistry of vimentin and E-cadherin expression in TRAIL-overexpressing EC1 cells and 
respective controls. NC, negative control. The data are presented as the mean \pm SEM of three independent experiments. ${ }^{\star \star \star} \mathrm{P}<0.001,{ }^{\star} * \mathrm{P}<0.01,{ }^{*} \mathrm{P}<0.05$ (paired t-test).
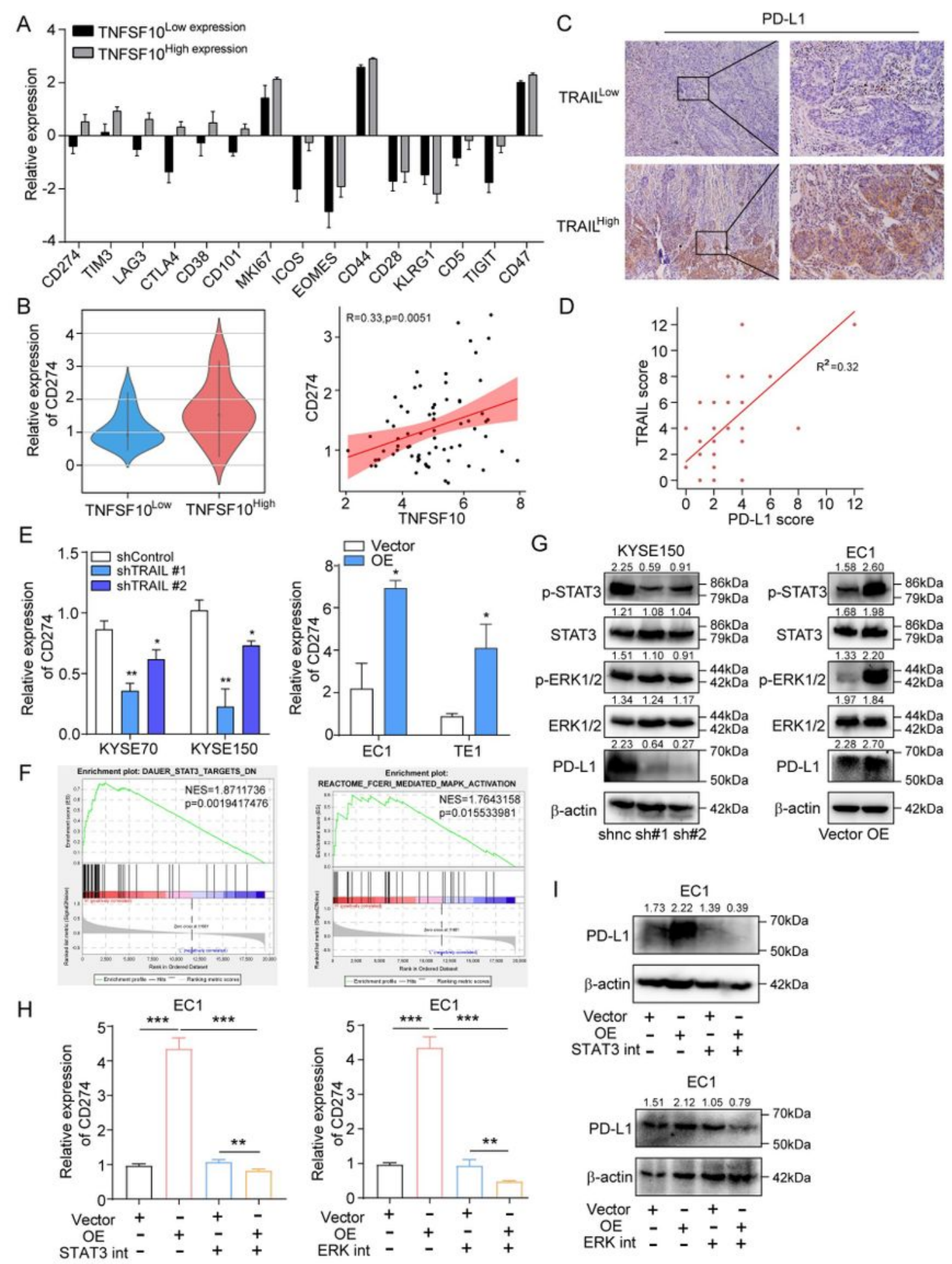

EC1

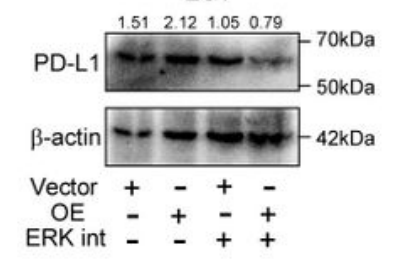

\section{Figure 5}

TRAIL promotes PD-L1 expression by upregulating the p-ERK/STAT3 signaling pathway. $(a, b)$ Analysis of TNFSF10 high- and low-expression genes; TNFSF10 and CD274 expression was significantly positively correlated in TCGA database. (c, d) TRAIL and PD-L1 expression was significantly positively correlated in 
human ESCC tissues ( $n=43$ ), as evidenced by immunohistochemistry. (e) RT-qPCR of CD274 mRNA levels in TRAIL-knockout (left) and overexpressed (right) ESCC cell lines. (f) KEGG enrichment analysis of differentially expressed genes. (g) Western blotting of STAT3, p-STAT3, ERK1/2, p-ERK1/2, PD-L1, and 》actin expression in TRAIL-knockout KYSE150 or TRAIL-overexpressed EC1. (h, i) RT-qPCR and western

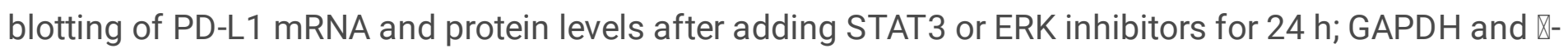
actin were used as control for RT-qPCR and western blotting, respectively.
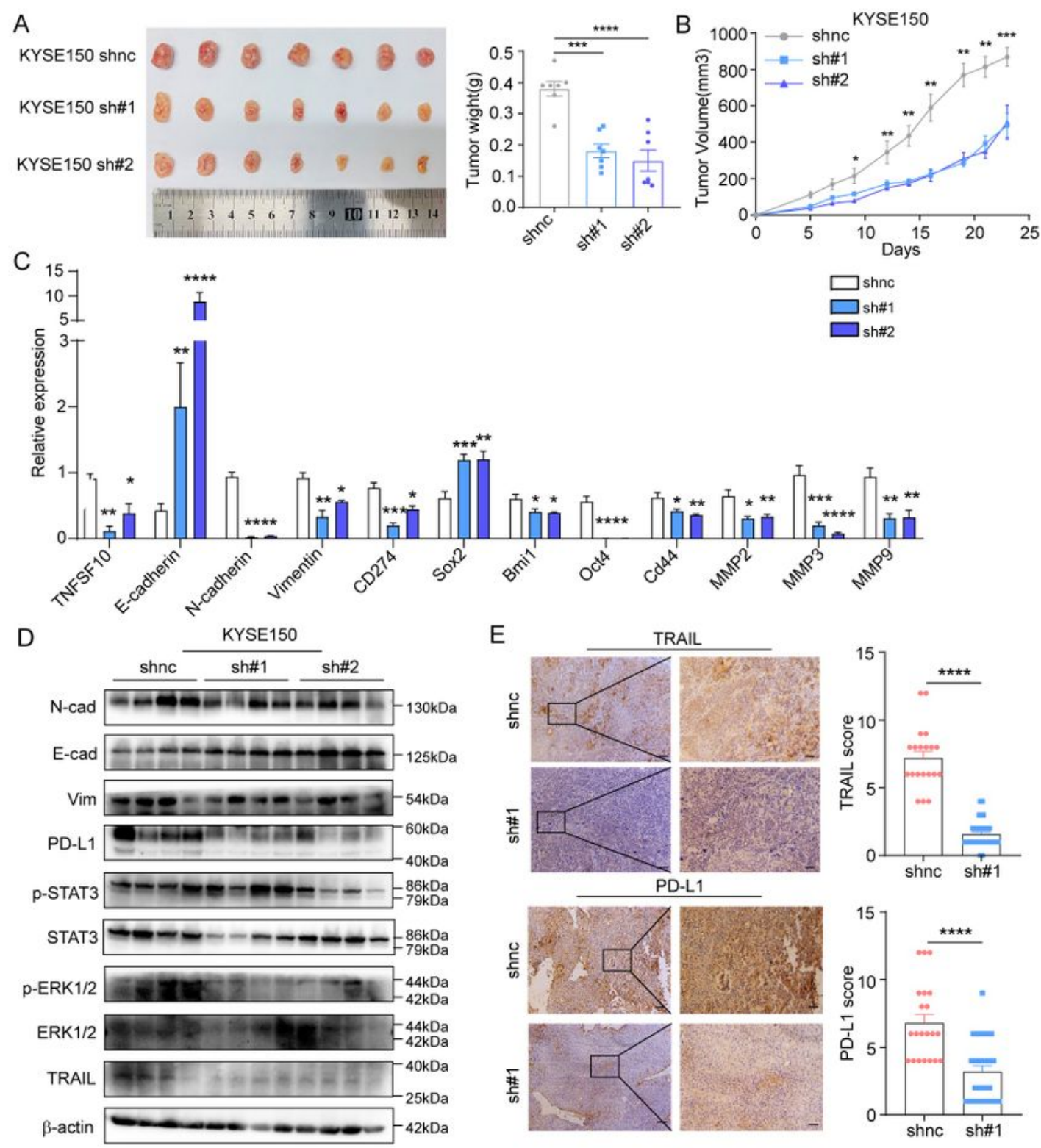

$\mathrm{F}$
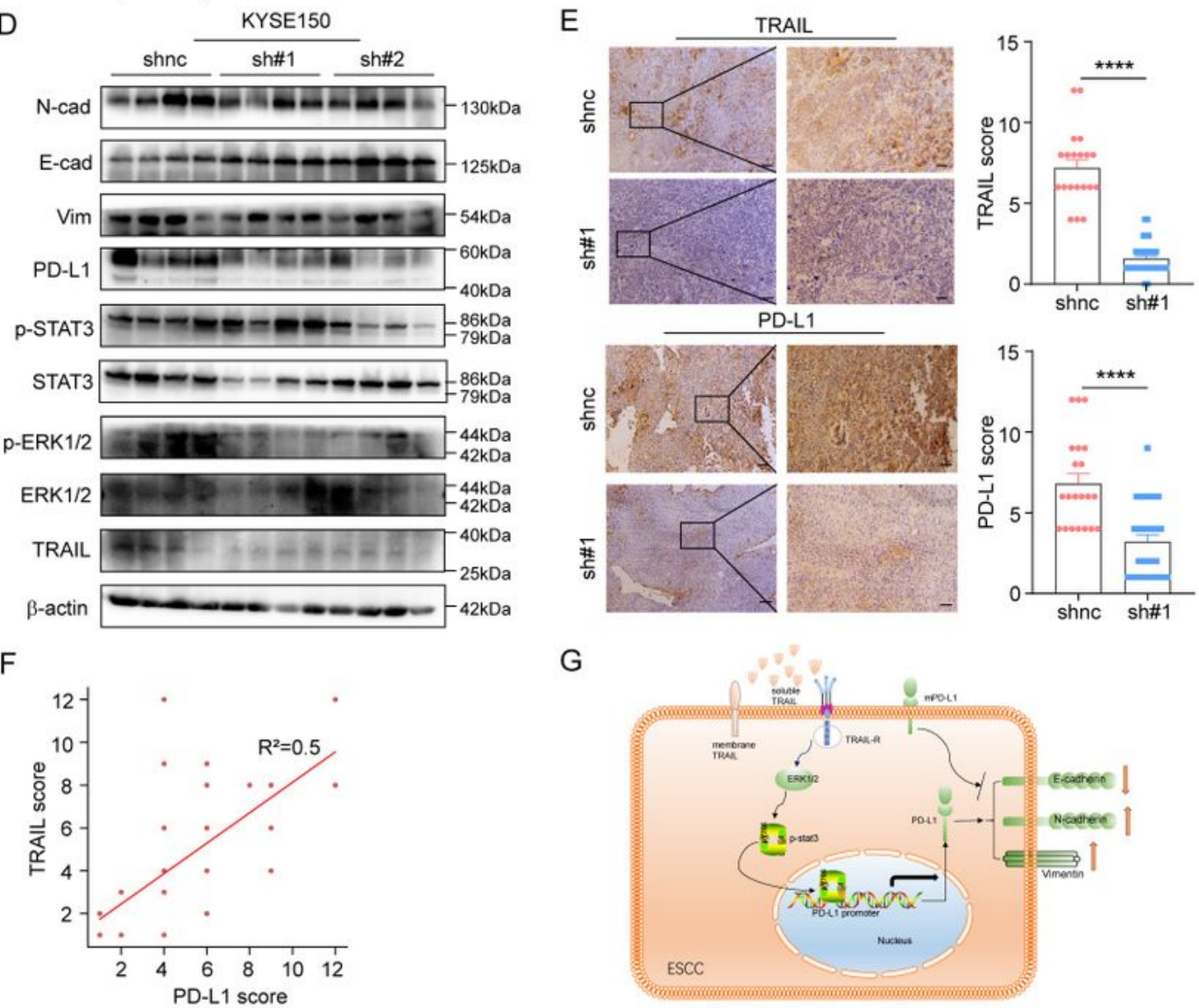

G

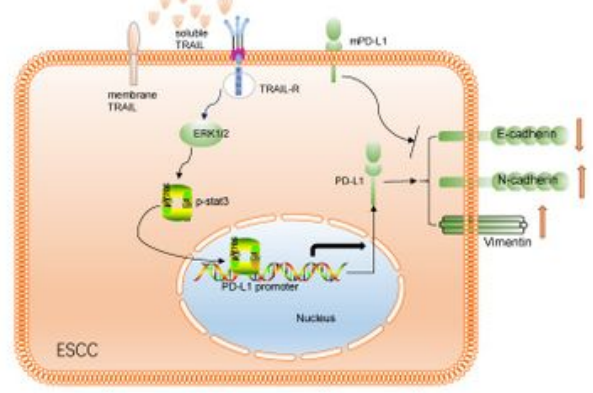

Figure 6 
TRAIL promotes EMT of ESCC via PD-L1 in vivo. $(a, b)$ Tumor weight and volume were monitored every other day, mice were sacrificed after 21 days. Tumor growth was significantly inhibited in TRAILknockdown cells. (c, d) mRNA and protein levels of EMT and stemness markers in mouse tumor tissues. (d) Knockdown of TRAIL downregulated STAT3 and ERK phosphorylation levels in tumor tissues. $(e, f)$ Immunohistochemistry of TRAIL and PD-L1 expression in animal tissues; immunohistochemical scoring and correlation analysis were performed. (g) TRAIL upregulated PD-L1 and promoted EMT progression in ECSS.

\section{Supplementary Files}

This is a list of supplementary files associated with this preprint. Click to download.

- SupplementaryTable1.docx

- SupplementaryFigures.docx 